\title{
Erratum to: Experimental analysis of the response of fresh wood stems subjected to localized impact loading
}

\author{
I. Olmedo ${ }^{1} \cdot$ F. Bourrier ${ }^{1}$ - D. Bertrand ${ }^{2}$. \\ D. Toe ${ }^{1} \cdot$ F. Berger ${ }^{1} \cdot$ A. Limam ${ }^{2}$
}

\section{Erratum to: Wood Sci Technol (2015) 49:623-646 DOI 10.1007/s00226-015-0713-0}

In the original publication of the article, Eqs. (13), (14) and (15) are incorrect. The correct versions of these equations are given below.

$$
\begin{gathered}
E_{\mathrm{b}}^{\mathrm{max}}=\frac{F_{\text {MaxDisp }}^{2} L^{3}}{96 \mathrm{MOE} I_{z}} \\
F_{\text {MaxDisp }}=v_{\text {in }} D^{2} \sqrt{\frac{3 \pi \mathrm{MOE} m_{\mathrm{eq}}}{4 L^{3}}} \\
y_{\max }=\frac{v_{\mathrm{in}}}{D^{2}} \sqrt{\frac{4 m_{\mathrm{eq}} L^{3}}{3 \pi \mathrm{MOE}}}
\end{gathered}
$$

The online version of the original article can be found under doi:10.1007/s00226-015-0713-0.

I. Olmedo

ignacio.olmedo@irstea.fr

1 Irstea Grenoble, UR EMGR, 2, rue de la papeterie, St. Martin d'Heres, France

2 INSA-Lyon (National Institut of Applied Sciences of Lyon), 20, Avenue Albert Einstein, 69621 Villeurbanne Cedex, France 